\title{
Patterns of deformations of Peregrine breather of order 3 and 4 solutions to the NLS equation with multi parameters
}

\author{
Pierre Gaillard $^{1} \cdot$ Mickaël Gastineau $^{2}$
}

Received: 28 September 2015/Accepted: 19 December 2015/Published online: 14 January 2016

(c) The Author(s) 2016. This article is published with open access at Springerlink.com

\begin{abstract}
In this article, one gives a classification of the solutions to the one dimensional nonlinear focusing Schrödinger equation (NLS) by considering the modulus of the solutions in the $(x, t)$ plan in the cases of orders 3 and 4 . For this, we use a representation of solutions to NLS equation as a quotient of two determinants by an exponential depending on $t$. This formulation gives in the case of the order 3 and 4, solutions with, respectively 4 and 6 parameters. With this method, beside Peregrine breathers, we construct all characteristic patterns for the modulus of solutions, like triangular configurations, ring and others.
\end{abstract}

Keywords NLS equation - Peregrine breathers - Rogue waves

\section{Introduction}

The rogue waves phenomenon currently exceed the strict framework of the study of ocean's waves [1-4] and play a significant role in other fields; in nonlinear optics [5, 6], BoseEinstein condensate [7], superfluid helium [8], atmosphere [9], plasmas [10], capillary phenomena [11] and even finance [12]. In the following, we consider the one-dimensional focusing nonlinear equation of Schrödinger (NLS) to describe the phenomena of rogue waves. The first results concerning the

Pierre Gaillard

pierre.gaillard@u-bourgogne.fr

Mickaël Gastineau

gastineau@imcce.fr

1 Université de Bourgogne, 9 Av. Alain Savary, Dijon, France

2 IMCCE, Observatoire de Paris, PSL Research University, CNRS, Sorbonne Universités UPMC Univ, Paris 06 Univ. Lille 77 Av. Denfert-Rochereau, 75014 Paris, France
NLS equation date from the Seventies. Precisely, in 1972 Zakharov and Shabat solved it using the inverse scattering method [13, 14]. The case of periodic and almost periodic algebro-geometric solutions to the focusing NLS equation was first constructed in 1976 by Its and Kotlyarov [15, 16]. The first quasi rational solutions of NLS equation were constructed in 1983 by Peregrine [17]. In 1986 Akhmediev, Eleonskii and Kulagin obtained the two-phase almost periodic solution to the NLS equation and obtained the first higher order analogue of the Peregrine breather [18-20]. Other analogues of the Peregrine breathers of order 3 and 4 were constructed in a series of articles by Akhmediev et al. [21-23] using Darboux transformations. The present paper presents multi-parametric families of quasi rational solutions of NLS of order $N$ in terms of determinants of order $2 N$ dependent on $2 N-2$ real parameters. The aim of this paper was to try to distinguish among all the possible configurations obtained by different choices of parameters, one those which have a characteristic in order to try to give a classification of these solutions.

\section{Expression of solu-tions of NLS equation in terms of a ratio of two determinants}

We consider the focusing NLS equation

$i v_{t}+v_{x x}+2|v|^{2} v=0$.

To solve this equation, we need to construct two types of functions $f_{j, k}$ and $g_{j, k}$ depending on many parameters. Because of the length of their expressions, one defines the functions $f_{v, \mu}$ and $g_{v, \mu}$ of argument $A_{v}$ and $B_{v}$ only in the appendix. We have already constructed solutions of equation NLS in terms of determinants of order $2 N$ which we call solution of order $N$ depending on $2 N-2$ real parameters. It is given in the following result [24-27]: 


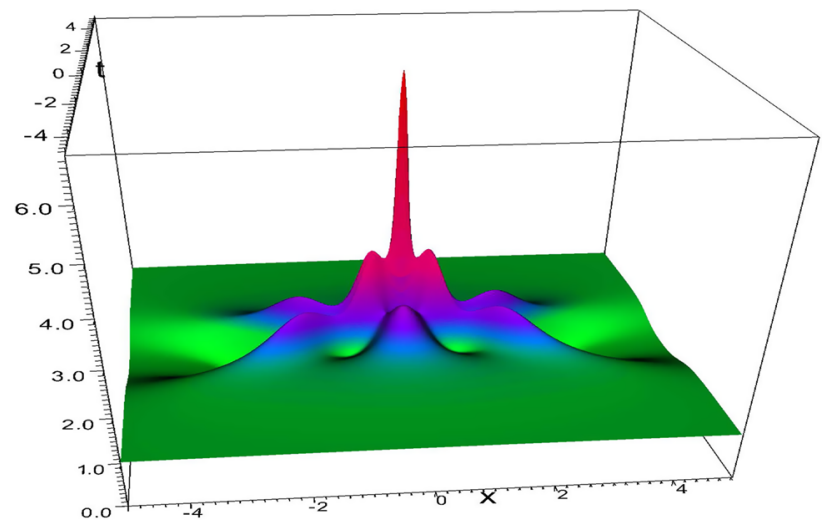

Fig. 1 Solution $P_{3}$ to NLS, $N=3, \tilde{a}_{1}=\tilde{b}_{1}=\tilde{a}_{2}=\tilde{b}_{2}=0$

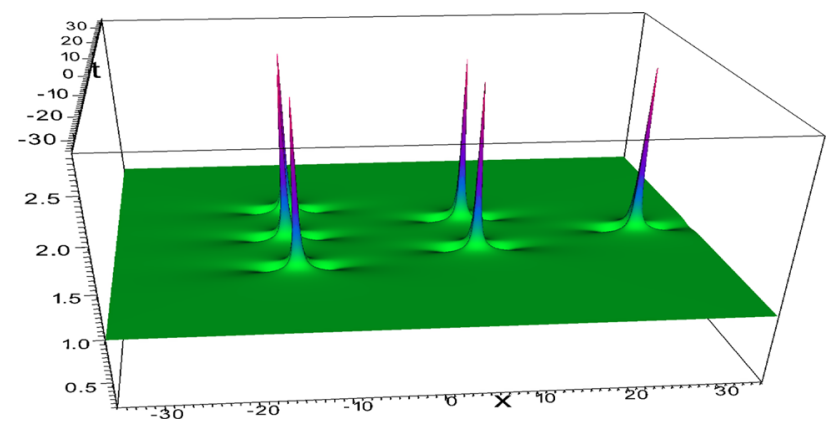

Fig. 2 Solution $1 T 6$ to NLS, $N=3, \tilde{a}_{1}=10^{4}, \tilde{b}_{1}=0, \tilde{a}_{2}=0, \tilde{b}_{2}=0$

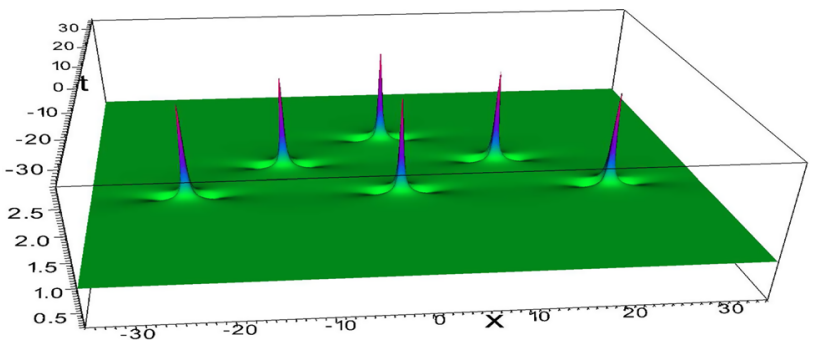

Fig. 3 Solution $1 T 6$ to NLS, $N=3, \tilde{a}_{1}=0, \tilde{b}_{1}=10^{4}, \tilde{a}_{2}=0, \tilde{b}_{2}=0$

Theorem 1 The functions $v$ defined by

$v(x, t)=\frac{\operatorname{det}\left(\left(n_{j k}\right)_{j, k \in[1,2 N]}\right)}{\operatorname{det}\left(\left(d_{j k}\right)_{j, k \in[1,2 N]}\right)} e^{2 i t-i \varphi}$

are quasi-rational solution of the NLS Eq. (1) depending on $2 N-2$ parameters $\tilde{a}_{j}, \tilde{b}_{j}, 1 \leq j \leq N-1$, where

$n_{j 1}=f_{j, 1}(x, t, 0)$,

$n_{j k}=\frac{\partial^{2 k-2} f_{j, 1}}{\partial \epsilon^{2 k-2}}(x, t, 0)$,

$n_{j N+1}=f_{j, N+1}(x, t, 0)$,

$n_{j N+k}=\frac{\partial^{2 k-2} f_{j, N+1}}{\partial \epsilon^{2 k-2}}(x, t, 0)$,

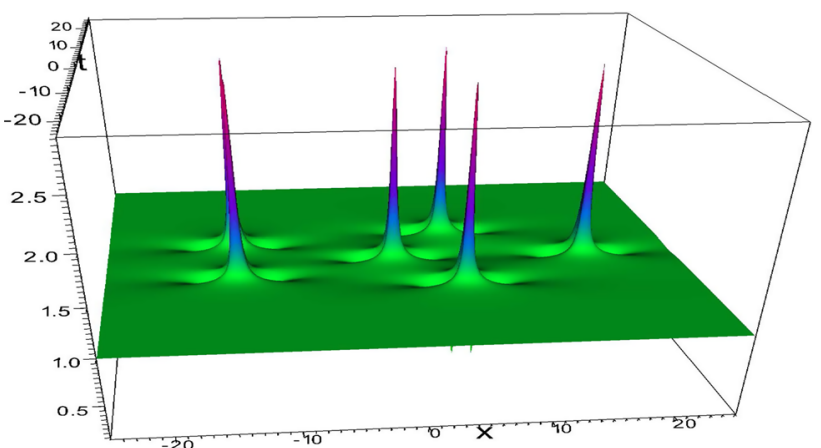

Fig. 4 Solution $1 R 5+1$ to NLS, $N=3, \tilde{a}_{1}=0, \tilde{b}_{1}=0, \tilde{a}_{2}=10^{6}$, $\tilde{b}_{2}=0$

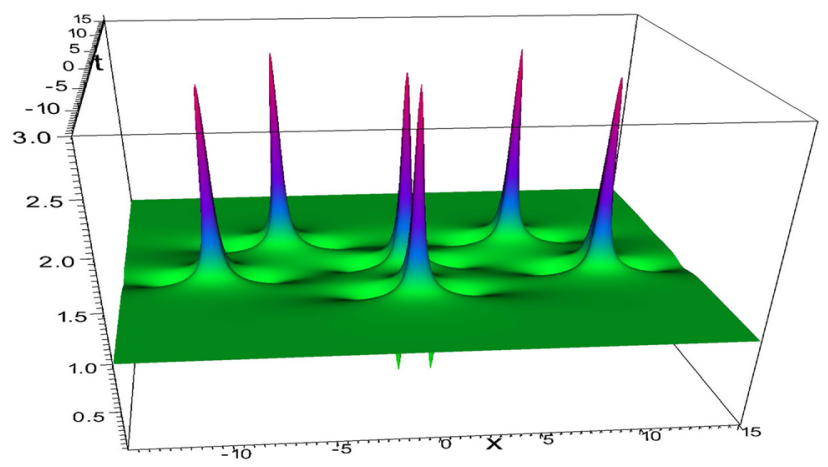

Fig. 5 Solution $1 R 5+1$ to NLS, $N=3, \tilde{a}_{1}=0, \tilde{b}_{1}=0, \tilde{a}_{2}=0$, $\tilde{b}_{2}=10^{5}$

$d_{j 1}=g_{j, 1}(x, t, 0)$,

$d_{j k}=\frac{\partial^{2 k-2} g_{j, 1}}{\partial \epsilon^{2 k-2}}(x, t, 0)$,

$d_{j N+1}=g_{j, N+1}(x, t, 0)$,

$d_{j N+k}=\frac{\partial^{2 k-2} g_{j, N+1}}{\partial \epsilon^{2 k-2}}(x, t, 0)$,

$2 \leq k \leq N, 1 \leq j \leq 2 N$

The functions $f$ and $g$ are defined in (9), (10), (11), (12).

\section{Patterns of quasi rational solutions to the NLS equation}

The solutions $v_{N}$ to NLS Eq. (2) of order $N$ depending on $2 N-2$ parameters $\tilde{a}_{j}, \tilde{b}_{j}$ (for $1 \leq j \leq N-1$ ) have been already explicitly constructed and can be written as

$v_{N}(x, t)=\frac{n(x, t)}{d(x, t)} \exp (2 i t)$ 


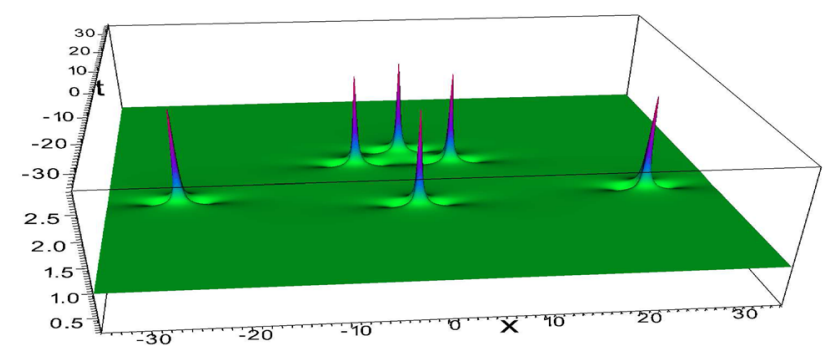

Fig. 6 Solution $1 A 3+1 T 3$ to NLS, $N=3, \tilde{a}_{1}=0, \tilde{b}_{1}=10^{4}, \tilde{a}_{2}=0$, $\tilde{b}_{2}=5 \times 10^{6}$

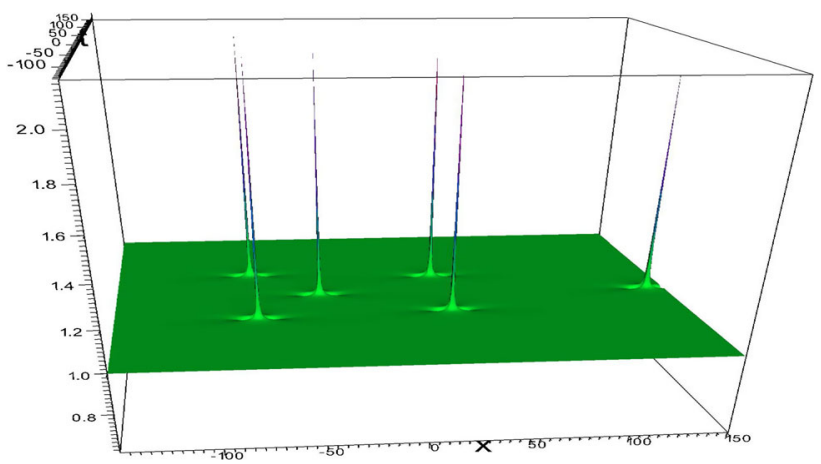

Fig. 7 Solution $1 A 3+1 T 3$ to NLS, $N=3, \quad \tilde{a}_{1}=10^{6}, \quad \tilde{b}_{1}=0$, $\tilde{a}_{2}=10^{10}, \tilde{b}_{2}=0$

$=\left(1-\alpha_{N} \frac{G_{N}(2 x, 4 t)+i H_{N}(2 x, 4 t)}{Q_{N}(2 x, 4 t)}\right) e^{2 i t}$

with

$$
\begin{aligned}
G_{N}(X, T) & =\sum_{k=0}^{N(N+1)} g_{k}(T) X^{k}, \\
H_{N}(X, T) & =\sum_{k=0}^{N(N+1} h_{k}(T) X^{k}, \\
Q_{N}(X, T) & =\sum_{k=0}^{N(N+1} q_{k}(T) X^{k} .
\end{aligned}
$$

For order 3 these expressions can be found in [28]; in the case of order 4, they can be found in [29]. In the following, based on these analytic expressions, we give a classification of these solutions by means of patterns of their modulus in the plane $(x ; t)$.

\section{Patterns of quasi-rational solutions of order 3 with 4 parameters}

\section{$P_{3}$ breather}

If we choose all parameters equal to $0, \tilde{a}_{1}=\tilde{b}_{1}=\ldots$ $=\tilde{a}_{N-1}=\tilde{b}_{N-1}=0$, we obtain the classical Peregrine breather given by

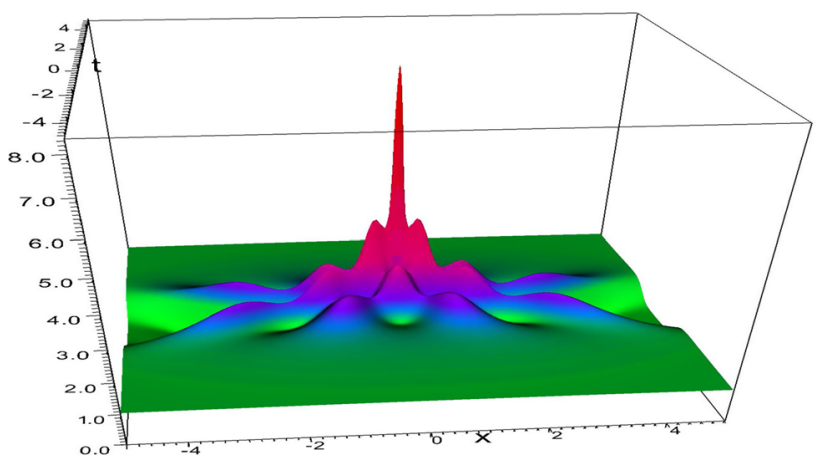

Fig. 8 Solution $P_{4}$ to NLS, $N=4, \tilde{a}_{1}=\tilde{b}_{1}=\tilde{a}_{2}=\tilde{b}_{2}=\tilde{a}_{3}=\tilde{b}_{3}=0$

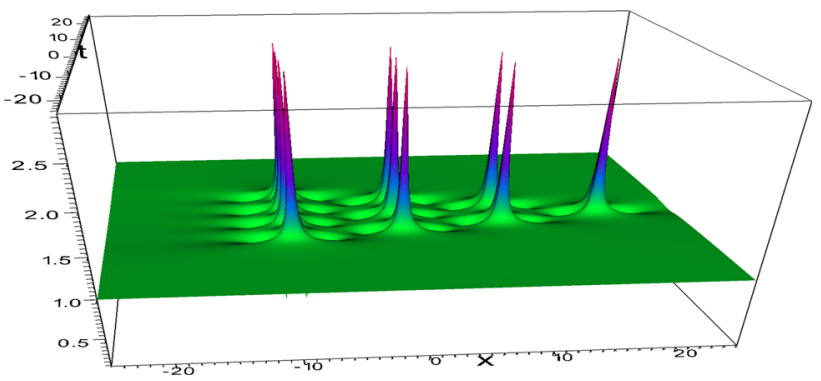

Fig. 9 Solution $1 T 10$ to NLS, $N=4, \tilde{a}_{1}=10^{3}, \tilde{b}_{1}=0, \tilde{a}_{2}=0, \tilde{b}_{2}=0$, $\tilde{a}_{3}=0, \tilde{b}_{3}=0$

\section{Triangles}

To shorten, the following notations are used: for example, the sequence $1 A 3+1 T 3$ means that the structure has one arc of 3 peaks and one triangle of 3 peaks. If we choose $\tilde{a}_{1}$ or $\tilde{b}_{1}$ not equal to 0 and all other parameters equal to 0 , we obtain triangular configuration with 6 peaks.

\section{Rings}

If we choose $\tilde{a}_{2}$ or $\tilde{b}_{2}$ not equal to 0 , all other parameters equal to 0 , we obtain ring configuration with peaks.

Arcs

If we choose $\tilde{a}_{1}$ and $\tilde{a}_{2}$ not equal to 0 and all other parameters equal to 0 (and vice versa, $\tilde{b}_{1}$ and $\tilde{b}_{2}$ not equal to 0 and all other parameters equal to 0 ), we obtain deformed triangular configuration which we can call as arc structure. 


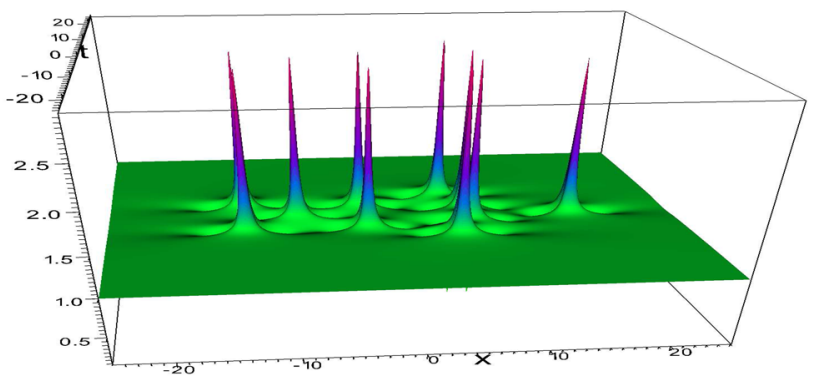

Fig. 10 Solution $2 R 5 / 5$ to NLS, $N=4, \tilde{a}_{1}=0, \tilde{b}_{1}=0, \tilde{a}_{2}=10^{5}$, $\tilde{b}_{2}=0, \tilde{a}_{3}=0, \tilde{b}_{3}=0$

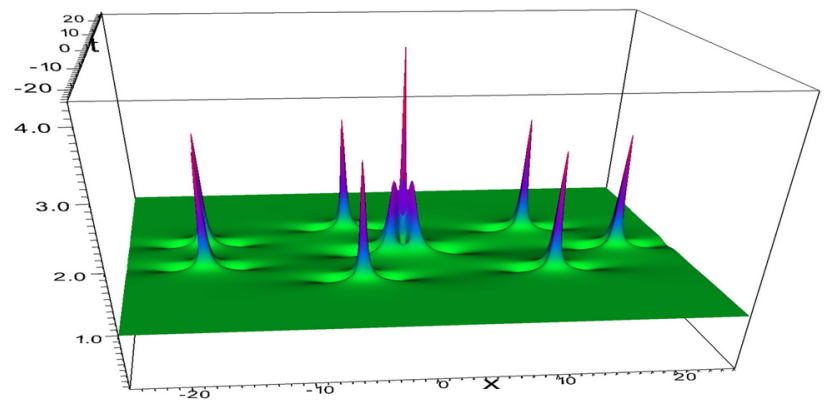

Fig. 11 Solution $1 R 7+P_{2}$ to NLS, $N=4, \tilde{a}_{1}=0, \tilde{b}_{1}=0, \tilde{a}_{2}=0$, $\tilde{b}_{2}=0, \tilde{a}_{3}=10^{8}, \tilde{b}_{3}=0$

\section{Patterns of quasi rational solutions of order 4 with 6 parameters}

\section{$P_{4}$ breather}

If we choose all parameters equal to 0 , $\tilde{a}_{1}=\tilde{b}_{1}=\ldots=\tilde{a}_{N-1}=\tilde{b}_{N-1}=0$, we obtain the classical Peregrine breather given in the following figure.

\section{Triangles}

To shorten, we use the notations defined in the previous section. If we choose $\tilde{a}_{1}$ or $\tilde{b}_{1}$ not equal to 0 and all other parameters equal to 0 , we obtain triangular configuration with 10 peaks.

Rings

If we choose $\tilde{a}_{2}$ or $\tilde{a}_{3}$ not equal to 0 and all other parameters equal to 0 (or vice versa $\tilde{b}_{2}$ or $\tilde{b}_{3}$ not equal to 0 and all other parameters equal to 0 ), we obtain ring configuration with 10 peaks.

\section{Arcs}

If we choose two parameters non equal to $0, \tilde{a}_{1}$ and $\tilde{a}_{2}$, or $\tilde{a}_{1}$ and $\tilde{a}_{3}$ not equal to 0 , or $\tilde{a}_{2}$ and $\tilde{a}_{3}$ and all other

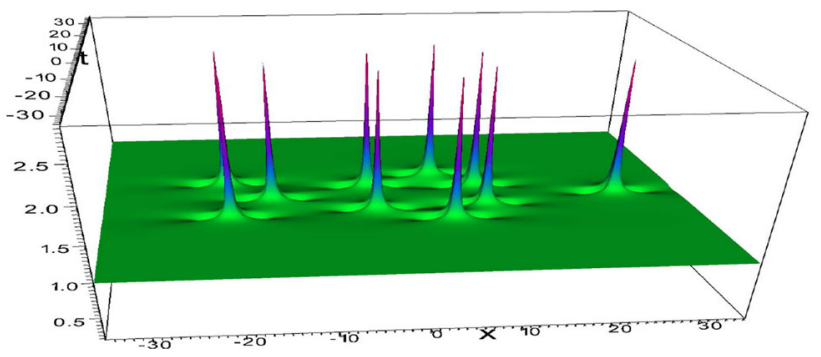

Fig. 12 Solution $2 A 3 / 4 I+T 3$ to NLS, $N=4, \tilde{a}_{1}=10^{3}, \tilde{b}_{1}=0$, $\tilde{a}_{2}=10^{6}, \tilde{b}_{2}=0, \tilde{a}_{3}=0, \tilde{b}_{3}=0$

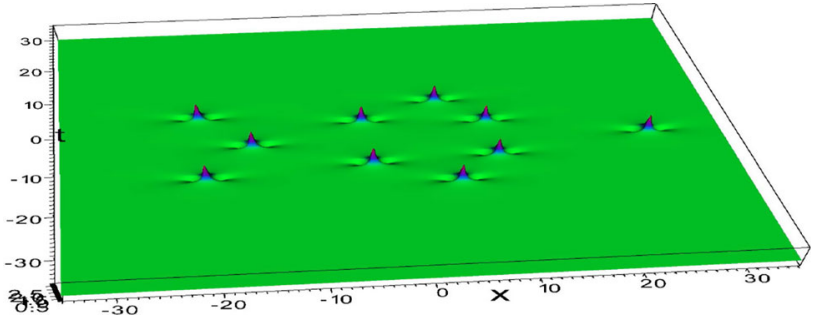

Fig. 13 Solution $2 A 3 / 4 I+T 3$ to NLS, $N=4, \quad \tilde{a}_{1}=10^{3}, \tilde{b}_{1}=0$, $\tilde{a}_{2}=10^{6}, \tilde{b}_{2}=0, \tilde{a}_{3}=0, \tilde{b}_{3}=0$, sight top

parameters equal to 0 (or vice versa for parameters $b$ ), we obtain arc configuration with 10 peaks. ${ }^{1}$

\section{Triangles inside rings}

If we choose three parameters non equal to $0, \tilde{a}_{1}, \tilde{a}_{2}$ and $\tilde{a}_{3}$ and all other parameters equal to 0 (or vice versa for parameters $b$ ), we obtain ring with inside triangle.

\section{Conclusion}

We have presented here patterns of modulus of solutions to the NLS focusing equation in the $(x, t)$ plane. This study can be useful at the same time for hydrodynamics as well for nonlinear optics; many applications in these fields have been realized, as it can be seen in recent works of Chabchoub et al. [30] or Kibler et al. [31]. This study tries to bring all possible types of patterns of quasi-rational solutions to the NLS equation. We see that we can obtain $2^{N-1}$ different structures at the order $N$. Parameters $a$ or $b$ give the same type of structure. For $a_{1} \neq 0$ (and other parameters equal to 0 ), we obtain triangular rogue wave; for $a_{j} \neq 0(j \neq 1$ and other parameters equal to 0$)$ we get ring rogue wave; in the other choices of parameters, we get in particular arc structures (or claw structure). This type of study has been realized in preceding works. Akhmediev

\footnotetext{
${ }^{1}$ In the following notations $2 A 4$ / 3I, I meaning Reversed.
} 


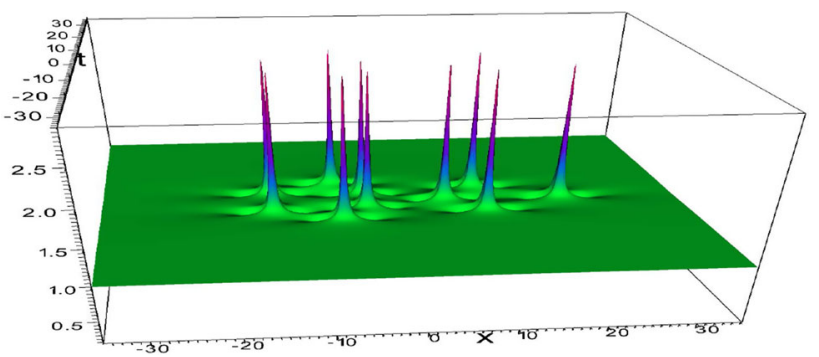

Fig. 14 Solution $2 A 4 / 3+1 T 3$ to NLS, $N=4, \tilde{a}_{1}=10^{3}, \tilde{b}_{1}=0$, $\tilde{a}_{2}=0, \tilde{b}_{2}=0, \tilde{a}_{3}=5 \times 10^{7}, \tilde{b}_{3}=0$

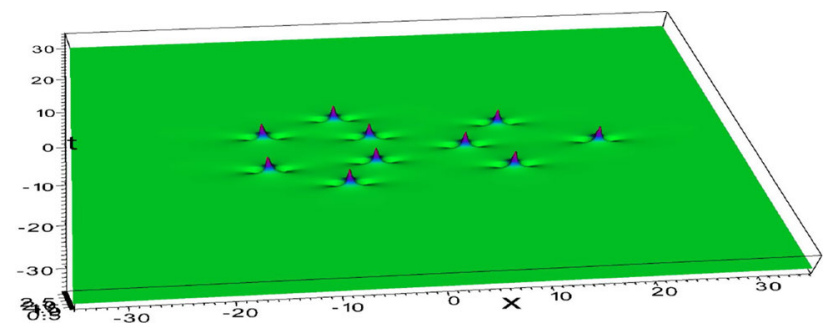

Fig. 15 Solution $2 A 4 / 3+1 T 3$ to NLS, $N=4, \tilde{a}_{1}=10^{3}, \quad \tilde{b}_{1}=0$, $\tilde{a}_{2}=0, \tilde{b}_{2}=0, \tilde{a}_{3}=5 \times 10^{7}, \tilde{b}_{3}=0$, sight top

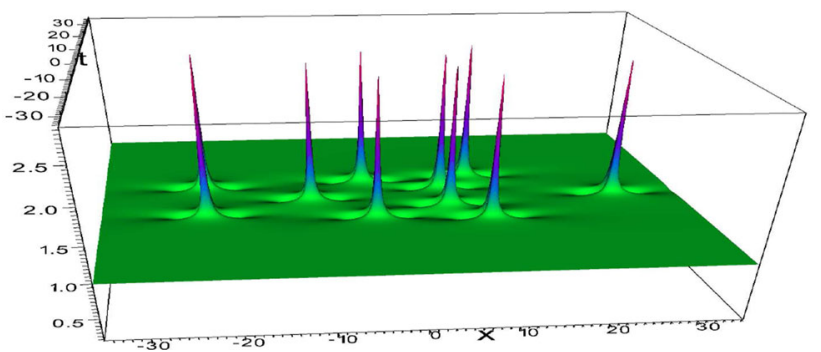

Fig. 16 Solution $2 A 3 / 4+1 T 3$ to NLS, $N=4, \quad \tilde{a}_{1}=0, \quad \tilde{b}_{1}=0$, $\tilde{a}_{2}=10^{6}, \tilde{b}_{2}=0, \tilde{a}_{3}=3 \times 10^{8}, \tilde{b}_{3}=0$

et al, study the order $N=2$ in [32], $N=3$ in [33]; the case $N=4$ was studied in particular $(N=5,6$ were also studied) in $[34,35]$ showing triangle and arc patterns; only one type of ring was presented. The extrapolation was done until the order $N=9$ in [36]. Ohta and Yang [37] presented the study of the case cas $N=3$ with rings and triangles. Recently, Ling and Zhao [38] presented the cases $N=$ 2, 3, 4 with rings, triangle and also claw structures.

In the present study, one sees appearing richer structures, in particular the appearance of a triangle of 3 peaks inside a ring of 7 peaks in the case of order $N=4$; to the best of my knowledge, it is the first time that this configuration for order 4 is presented. In this way, we try to bring a better understanding to the hierarchy of NLS rogue wave solutions. It will be relevant to go on this study with higher orders.

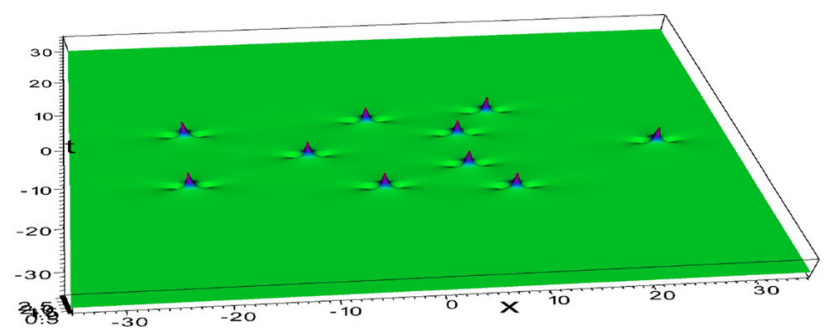

Fig. 17 Solution $2 A 3 / 4+1 T 3$ to NLS, $N=4, \quad \tilde{a}_{1}=0, \quad \tilde{b}_{1}=0$, $\tilde{a}_{2}=10^{6}, \tilde{b}_{2}=0, \tilde{a}_{3}=3 \times 10^{8}, \tilde{b}_{3}=0$, sight top

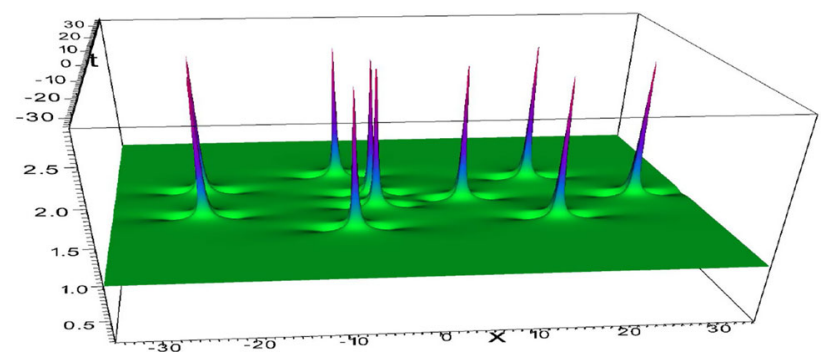

Fig. 18 Solution $1 A 7+1 T 3$ to NLS, $N=4, \quad \tilde{a}_{1}=10^{3}, \quad \tilde{b}_{1}=0$, $\tilde{a}_{2}=10^{3}, \tilde{b}_{2}=0, \tilde{a}_{3}=10^{9}, \tilde{b}_{3}=0$

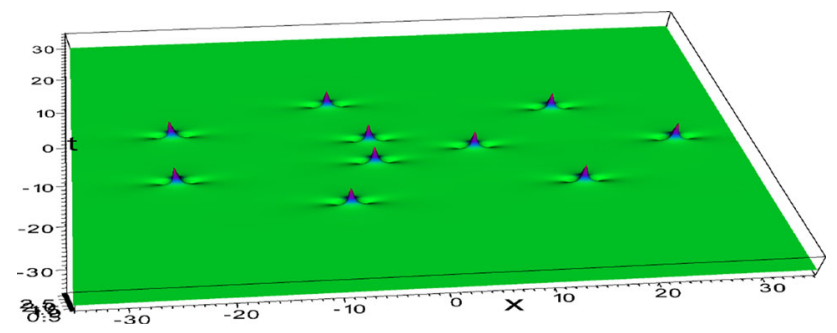

Fig. 19 Solution $1 A 7+1 T 3$ to NLS, $N=4, \quad \tilde{a}_{1}=10^{3}, \quad \tilde{b}_{1}=0$, $\tilde{a}_{2}=10^{3}, \tilde{b}_{2}=0, \tilde{a}_{3}=10^{9}, \tilde{b}_{3}=0$, sight top

Open Access This article is distributed under the terms of the Creative Commons Attribution 4.0 International License (http://creative commons.org/licenses/by/4.0/), which permits unrestricted use, distribution, and reproduction in any medium, provided you give appropriate credit to the original author(s) and the source, provide a link to the Creative Commons license, and indicate if changes were made.

\section{Appendix: Parameters and functions}

We consider the terms $\lambda_{v}$ satisfying the relations for $1 \leq j \leq N$

$0<\lambda_{j}<1, \lambda_{N+j}=-\lambda_{j}$,

$\lambda_{j}=1-2 \epsilon^{2} j^{2}$,

with $\epsilon$ a small number intended to tend towards 0 . The terms $\kappa_{v}, \delta_{v}, \gamma_{v}$ are functions of the parameters $\lambda_{v}$, 
$1 \leq v \leq 2 N$. They are given by the following equations, for $1 \leq j \leq N$ :

$$
\begin{aligned}
& \kappa_{j}=2 \sqrt{1-\lambda_{j}^{2}}, \quad \delta_{j}=\kappa_{j} \lambda_{j}, \\
& \gamma_{j}=\sqrt{\frac{1-\lambda_{j}}{1+\lambda_{j}}} \\
& \kappa_{N+j}=\kappa_{j}, \quad \delta_{N+j}=-\delta_{j}, \\
& \gamma_{N+j}=1 / \gamma_{j} .
\end{aligned}
$$

The terms $x_{r, v} r=3,1$ are defined by

$$
\begin{aligned}
& x_{r, j}=(r-1) \ln \frac{\gamma_{j}-i}{\gamma_{j}+i}, \\
& x_{r, N+j}=(r-1) \ln \frac{\gamma_{N+j}-i}{\gamma_{N+j}+i} .
\end{aligned}
$$

The parameters $e_{v}$ are given by

$e_{j}=i a_{j}-b_{j}, \quad e_{N+j}=i a_{j}+b_{j}$,

where $a_{j}$ and $b_{j}$ are chosen in the form

$$
\begin{aligned}
& a_{j}=\sum_{k=1}^{N-1} \tilde{a_{k}} \epsilon^{2 k+1} j^{2 k+1}, \\
& b_{j}=\sum_{k=1}^{N-1} \tilde{b_{k}} \epsilon^{2 k+1} j^{2 k+1}, \\
& 1 \leq j \leq N,
\end{aligned}
$$

with $\tilde{a_{j}}, \tilde{b_{j}}, 1 \leq j \leq N-1,2 N-2$, arbitrary real numbers.

The functions $f_{v, 1}$ and $g_{v, 1}, 1 \leq v \leq N$ are defined by

$$
\begin{aligned}
& f_{4 j+1,1}=\gamma_{k}^{4 j-1} \sin A_{1}, \\
& f_{4 j+2,1}=\gamma_{k}^{4 j} \cos A_{1}, \\
& f_{4 j+3,1}=-\gamma_{k}^{4 j+1} \sin A_{1}, \\
& f_{4 j+4,1}=-\gamma_{k}^{4 j+2} \cos A_{1}, \\
& f_{4 j+1, N+1}=\gamma_{k}^{2 N-4 j-2} \cos A_{N+1}, \\
& f_{4 j+2, N+1}=-\gamma_{k}^{2 N-4 j-3} \sin A_{N+1}, \\
& f_{4 j+3, N+1}=-\gamma_{k}^{2 N-4 j-4} \cos A_{N+1}, \\
& f_{4 j+4, N+1}=\gamma_{k}^{2 N-4 j-5} \sin A_{N+1}, \\
& g_{4 j+1,1}=\gamma_{k}^{4 j-1} \sin B_{1}, \\
& g_{4 j+2,1}=\gamma_{k}^{4 j} \cos B_{1}, \\
& g_{4 j+3,1}=-\gamma_{k}^{4 j+1} \sin B_{1}, \\
& g_{4 j+4,1}=-\gamma_{k}^{4 j+2} \cos B_{1}, \\
& g_{4 j+1, N+1}=\gamma_{k}^{2 N-4 j-2} \cos B_{N+1}, \\
& g_{4 j+2, N+1}=-\gamma_{k}^{2 N-4 j-3} \sin B_{N+1}, \\
& g_{4 j+3, N+1}=-\gamma_{k}^{2 N-4 j-4} \cos B_{N+1}, \\
& g_{4 j+4, N+1}=\gamma_{k}^{2 N-4 j-5} \sin B_{N+1} .
\end{aligned}
$$

The arguments $A_{v}$ and $B_{v}$ of these functions are defined by $1 \leq v \leq 2 N$ :

$$
\begin{aligned}
& A_{v}=\kappa_{v} x / 2+i \delta_{v} t-i x_{3, v} / 2-i e_{v} / 2 \\
& B_{v}=\kappa_{v} x / 2+i \delta_{v} t-i x_{1, v} / 2-i e_{v} / 2
\end{aligned}
$$

\section{References}

1. Kharif, C., Pelinovsky, E., Slunyaev, A.: Rogue waves in the Ocean. Springer (2009)

2. Akhmediev, N., Pelinovsky, E.: Discussion and debate: rogue waves - towards a unifying concept? Eur. Phys. Jour. Spec. Top. V 185 (2010)

3. Kharif, Ch., Pelinovsky, E.: Physical mechanisms of the rogue wave phenomenon. Eur. Jour. Mech. B Fluid 22(6), 603-634 (2003)

4. Slunyaev, A., Didenkulova, I., Pelinovsky, E.: Rogue waters. Cont. Phys. 52(6), 571-590 (2003)

5. Solli, D.R., Ropers, C., Koonath, P., Jalali, B.: Optical rogue waves. Nature 450, 1054-1057 (2007)

6. Dudley, J.M., Genty, G., Eggleton, B.J.: Optical rogue waves. Opt. Express 16, 3644 (2008)

7. Bludov, Y.V., Konotop, V.V., Akhmediev, N.: Matter rogue waves. Phys. Rev. A 80(033610), 1-5 (2009)

8. Ganshin, A.N., Efimov, V.B., Kolmakov, G.V., Mezhov-Deglin, L.P., McClintok, P.V.E.: Observation of an inverse energy cascade in developed acoustic turbulence in superfluid helium. Phys. Rev. Lett. 101, 065303 (2008)

9. Stenflo, L., Marklund, M.: Rogue waves in the atmosphere. J. Plasma Phys. 76(3-4), 293-295 (2010)

10. Ruderman, M.S.: Freak waves in laboratory and space plasmas. Eur. Phys. J. Spec. Top. 185, 57-66 (2010)

11. Shats, M., Punzman, H., Xia, H.: Matter rogue waves. Phys. Rev. Lett. 104(104503), 1-5 (2010)

12. Yan, Z.Y.: Financial rogue waves. Commun. Theor. Phys. V 54, $5,947,1-4,(2010)$

13. Zakharov, V.E.: Stability of periodic waves of finite amplitude on a surface of a deep fluid. J. Appl. Tech. Phys 9, 86-94 (1968)

14. Zakharov, V.E., Shabat, A.B.: Exact theory of two dimensional self focusing and one dimensinal self modulation of waves in nonlinear media. Sov. Phys. JETP 34, 62-69 (1972)

15. Its, A.R., Kotlyarov, V.P.: Explicit expressions for the solutions of nonlinear Schrödinger equation. Dockl. Akad. Nauk. SSSR S A V 965, 11 (1976)

16. Its, A.R., Rybin, A.V., Salle, M.A.: Exact integration of nonlinear Schrödinger equation. Teore. i Mat. Fiz. 74(1), 29-45 (1988)

17. Peregrine, D.: Water waves, nonlinear Schrödinger equations and their solutions. J. Aust. Math. Soc. Ser. B 25, 16-43 (1983)

18. Akhmediev, N., Eleonsky, V., Kulagin, N.: Generation of periodic trains of picosecond pulses in an optical fiber: exact solutions. Sov. Phys. JETP V 62, 894-899 (1985)

19. Eleonskii, V., Krichever, I., Kulagin, N.: Rational multisoliton solutions to the NLS equation, Soviet Doklady 1986 sect. Math. Phys. 287, 606-610 (1986)

20. Akhmediev, N., Eleonskii, V., Kulagin, N.: Exact first order solutions of the nonlinear Schrödinger equation. Theor. Math. Phys. 72(2), 183-196 (1987)

21. Akhmediev, N., Ankiewicz, A., Soto-Crespo, J.M.: Rogue waves and rational solutions of nonlinear Schrödinger equation. Phys. Rev. E 80(026601), 1-9 (2009)

22. Akhmediev, N., Ankiewicz, A., Clarkson, P.A.: Rogue waves, rational solutions, the patterns of their zeros and integral relations. J. Phys. A Math. Theor. 43(122002), 1-9 (2010) 
23. Chabchoub, A., Hoffmann, H., Onorato, M., Akhmediev, N.: Super rogue waves: observation of a higher-order breather in water waves. Phys. Rev. X 2(011015), 1-6 (2012)

24. Gaillard, P.: Families of quasi-rational solutions of the NLS equation and multi-rogue waves. J. Phys. A Meth. Theor. 44, 1-15 (2011)

25. Gaillard, P.: Wronskian representation of solutions of the NLS equation and higher Peregrine breathers. J. Math. Sci. Adv. Appl. 13(2), 71-153 (2012)

26. Gaillard, P.: Degenerate determinant representation of solution of the NLS equation, higher Peregrine breathers and multi-rogue waves. J. Math. Phys. V 54, 013504-1-32 (2013)

27. Gaillard, P.: Multi-parametric deformations of the Peregrine breather of order $\mathrm{N}$ solutions to the NLS equation and multirogue waves. Adv. Res. 4, 346-364 (2015)

28. Gaillard, P.: Deformations of third order Peregrine breather solutions of the NLS equation with four parameters. Phys. Rev. E V 88, 042903-1-9 (2013)

29. Gaillard, P.: Six-parameters deformations of fourth order Peregrine breather solutions of the NLS equation. J. Math. Phys. V 54, 073519-1-22 (2013)

30. Chabchoub, A., Hoffmann, N.P., Akhmediev, N.: Rogue wave observation in a water wave tank, Phys. Rev. Lett. V 106, 204502-1-4 (2011)
31. Kibler, B., Fatome, J., Finot, C., Millot, G., Dias, F., Genty, G., Akhmediev, N., Dudley, J.M.: The Peregrine soliton in nonlinear fibre optics. Nat. Phys. 6, 790-795 (2010)

32. Ankiewicz, A., Kedziora, D.J., Akhmediev, N.: Rogue wave triplets. Phys Lett. A 375, 2782-2785 (2011)

33. Kedziora, D.J., Ankiewicz, A., Akhmediev, N.: The phase patterns of higher-order rogue waves. J. Opt. V 15, 064011-1-9 (2013)

34. Kedziora, D.J., Ankiewicz, A., Akhmediev, N.: Circular rogue wave clusters. Phys. Rev. E 84(056611), 1-7 (2011)

35. Kedziora, D.J., Ankiewicz, A., Akhmediev, N.: Triangular rogue wave cascades. Phys. Rev. E 86(056602), 1-9 (2012)

36. Kedziora, D.J., Ankiewicz, A., Akhmediev, N.: Classifying the hierarchy of the nonlinear Schrödinger equation rogue waves solutions. Phys. Rev. E V 88, 013207-1-12 (2013)

37. Ohta, Y., Yang, J.: General high-order rogue waves and their dynamics in the nonlinear Schrödinger equation. Proc. R. Soc. A 468(2142), 1716-1740 (2012)

38. Ling, L., Zhao, L.C.: Simple determinant representation for rogue waves of the nonlinear Shrodinger equation. Phys. Rev. E V 88, 043201-1-9 (2013) 